\begin{tabular}{ccc}
\hline International Journal of Engineering \& Technology, $7(3.30)(2018) 90-95$ \\
SPC \\
Website $:$ www.sciencepubco.com/index.php/IJET \\
Research paper
\end{tabular}

\title{
Three Dimensional Cube
}

\author{
Pushpa Gothwal $^{1}$, Sandesh Singh Shekhawat ${ }^{2}$ \\ ${ }^{1}$ Amity University Rajasthan \\ ${ }^{2}$ Amity School of Engineering and technology, dep. of ECE \\ *Corresponding author E-mail: pushpaprof@gmail.com
}

\begin{abstract}
Three dimensional cube is the 3D display device implemented using RGB LED. The objective of this paper to design a 3D CUBE using shift registers controlled by the microcontroller. It consists of using a 8-bit microcontrollers (Arduino Mega 2560, Arduino Uno and AT89s52) with on chip ADC, PWM and UART, RGB LEDs, pulse sensor (SEN-11574), shift registers (74HC595N) as core element of the system. It has six keys used for selecting the defined patterns like square, cube, heart traingle etc.. The application of this project is used for entertainment, display the measured physiological parameter (Heart beat), learning and teaching purpose. It can also help to medical professionals for teaching purpose, defence personals in RADAR and architects.
\end{abstract}

Keywords: Cube, Microcontroller, Display, RGB.

\section{Introduction}

In this modern and high tech era of human kind, technology is growing rapidly. In the field of electronics, new research and development has made this field a revolution. Nowadays, every hum an being on this planet is surrounded by electronic gadgets like mobile, laptops, television, games etc. But all these devices are useless if they do not have a display device attached to it. The result of our instructions cannot be seen without a display device. So display devices become a major part of any electronic circuit.Various display devices have been formulated over the years in the form of LEDs, Seven Segment Displays, LCDs, Plasma displays, etc. These displays can either show data in 1D or 2D at maximum but none of them can show data in a 3D format. Several 3D cubes have been designed earlier, like 3D LED Cube, True 3D Image Manipulator, A Volumetric 3D LED Display, etc. They are made for 3D animations, interaction with external stimulus like accelerometer, audio waves etc. So, this paper aims to show the same form of data in a 3D format by using lattice structure of RGB LEDs. The sequence of array matrix is kept in such a way that it forms a cube. Hence it forms an $8 \times 8 \times 8$ RGB LED cube. The ability to visualize the data through all the 3 dimensions (length, breadth and height) gives this display the name "Volumetric Display".

\section{Design Approach}

The hardware setup as shown in figure 1 consists of following components:

$\begin{array}{ll}\text { - } & \text { Main 8x8x8 3D RGB LED cube } \\ \text { - } & \text { Shift Registers } \\ \text { - } & \text { Interfoller } \\ & \text { Inteing Keys, and }\end{array}$

\subsection{Heart Beat Circuit Interfaced With the Main Con- troller.}

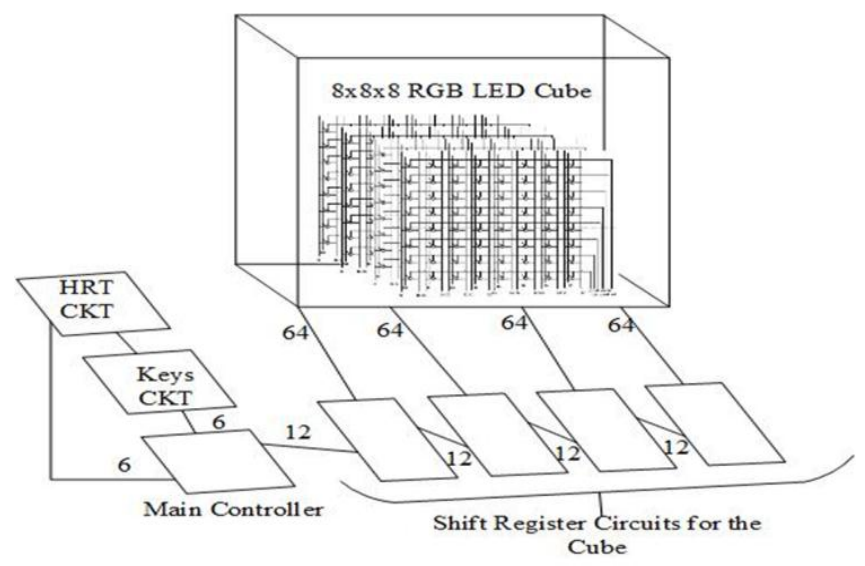

Fig .1: Hardware setup

The whole circuitry is designed in such a way that cube does not show any display until a key is interfaced with the main controller. There are basically six keys which are interfaced with the controller. Key- 1 activates the heart beat circuit. The heart beat circuit shows the heart rate of a human being in one minute. According to the heart rate, different colour LEDs glow and show different patterns according to the heart beat of the person. The second Key is used to glow the whole cube in green colour. The third key is used to show various study patterns like square, horizontal lines, vertical lines, point in a space, different corners of a cube, etc. This can help many students understand basic as well as complex concepts of 1D, 2D and 3D. The fourth key is also used to show study patterns. In this various horizontal and vertical lines are shown in different planes. This explains the basic concepts of parallel lines, collinear lines, parallel but not collinear lines etc. 
The fifth key is used to turn off all the LEDs of the cube. This is created to initialize the cube to its starting condition. The sixth key is used to show various LED patterns for entertainment purposes.The Main controller is the brain of the system which regulates all the functioning of the cube. Whatever is happening in the cube is due to the main controller. The next part in the instrument setup is shift register board which store and send various data to LEDs according to the command given by the main controller. The $8 \times 8 \times 8$ RGB LED cube is the main and the most important part of the whole instrument setup. It consists of 512 RGB LEDs. All the outputs of various inputs given by the keys are shown on it. It generates various LED patterns as its output.

\section{Block Diagram of Main Cube}

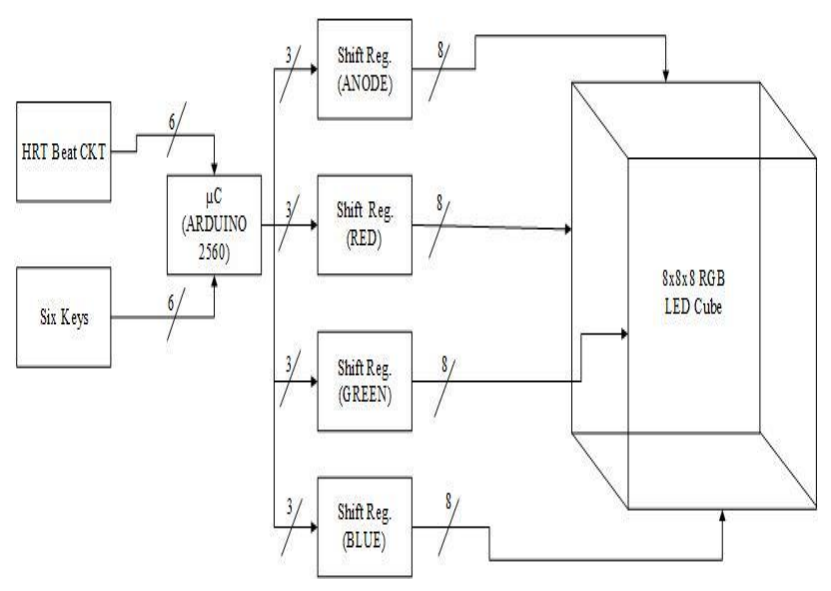

Fig. 2: Block diagram of main cube

The block diagram of main cube as shown in figure 2 . In this, left most part of the block diagram represents the input part of the system. In the middle, we have the controller and processing part and the right most part of the block diagram represents the output of the overall system. There are overall 12 inputs which are fed to the microcontroller. Six inputs come from the six keys which are interfaced with microcontroller and other six comes from the heart beat circuit. The six keys are the most important part of this system since nothing happens in the cube until a key is pressed and interfaced with the controller. It represents and functions in the following manner:

KEY 1 : Activates the heart beat circuit.

KEY 2 : Whole cube glows in green color.

KEY 3 : Generates the 2nd study patterns

KEY 4 : Generates the 1st study patterns

KEY 5 : Turn off all the LEDs of the cube.

KEY 6 : Shows various entertainment patterns

The next part of the block diagram is the Controller. It is the most important part of the whole system since it is the brain of the system. The main Micro Controller used in this project is Arduino Mega 2560.The six inputs from keys and six inputs from the heart beat circuit are fed to the digital pins of Arduino. The digital pins can have only two values either 'High' (1) or 'Low' (0). When a key is pressed, one of the digital pin of Arduino becomes high (1) When such a condition occurs, the program dumped in the Arduino tells the controller which all pins controlling the outputs should become high or low. Accordingly the output is generated on the output device. Similarly, when the conditions of other inputs are met, output controlling pins of controller react in accordance with it. Arduino Mega (2560) sends out 12 outputs which are fed to four shift registers ( 3 output pins connected to each register IC).
The 12 digital outputs pins of the micro-controller are sending data to 4 shift registers (74HC595N). Each shift register gets connected to three digital output pins from the micro-controller. The three pins: Data, Clock and Latch pins of the shift registers are connected to the micro controller. Here 4 shift registers are used to send data bytes to anode, red, green and blue terminals of RGB LEDs. These data bytes are sent in accordance to the input conditions and instructions from the micro controller. Eight shift registers are serially connected to one another to control one of the terminals of RGB LEDs.

The final part of the block diagram represents the output device which is the 3D RGB LED cube. All the outputs of processing in microcontroller are shown on it. The $8-$ bit data from the shift registers (for anode, red, green and blue) are sent to the terminals of the RGB LED Cube. According to the data from shift registers, anode; red; green and blue terminals of RGB Led become high (1) or low (0) and thus form the required patterns for the given input.

\section{Instrument Hardware}

Instrument Hardware is the collection of physical elements that constitutes the system of this project. The main parts of the hardware are the input devices, processing unit, memory and the output devices. The input devices used in this project are switches and heart pulse from the pulse sensor. Processing part is in the form of micro controllers (Arduino Mega 2560, Arduino UNO andAT89s52) and shift registers (74HC595N). The output devices used in this project are LCD and the 3D RGB LED cube. LCD shows the heart rate of the subject and the RGB LED cube is used to show various LED patterns according to the given input.

The main cube functions on the basis of instruction given through different switches (or keys) as shown in figure 3 and 4 . There are basically six switches used to activate the cube in different ways. As soon as a switch is pressed, the corresponding pin of the micro controller (Arduino Mega 2560) becomes high. Now, the program fed in the micro controller comes into action. According to the given input it makes the latch pins of the entire shift registers low. Then on the rising edge of the clock pulse sent by the micro controller to the shift register, required data is stored inside the shift register. A shift register can store only 8 bit data. When a data sent is more than a byte, the previous data gets shifted to the next shift register which is connected serially to the first one. So in this way we can control ' $n$ ' no. of RGB LED terminals and we only need to use 3 digital pins of the cube controller.

Another part of this whole system is the heart beat circuit. It is interfaced with the cube controller as input device. The input to the heart beat circuit is the heart pulse of the subject from the pulse sensor. The data we get from the pulse sensor is in the form of analog signal. For further processing of this signal, we first need to convert it into digital signals. This is done through ADC of Arduino UNO micro controller. After getting the required digital signals, some part of it is sent to Arduino Mega 2560 (cube controller) and rest of it is sent to AT89s52. In the following circuit we have used two AT89s52 micro controllers. The first micro controller is used to generate a time delay of one minute and to control the gate of next controller for one minute. The one minute time delay is shown through a LED. The next AT89s52 is the master controller for the heart beat circuit. The heart pulse is connected to it at P3.5 and the gate one minute time delay signal from first controller is connected to it at P3.3. as external hardware interrupt. The master controller accordingly counts the edges of the heart pulse for one minute and shows the result on a LCD screen. According to the heart rate of the subject, five P2 port pins of the master controller are made high or low. These 5 pins of P2 port are connected with cube controller (Arduino 2560). It tells the cube controller to glow blue LEDs of the cube when heart rate is less than 60, Green + Blue LEDs of cube when heart rate is in between 60 to 70, Green LEDs when heart rate is in between 70 to 
80, Green + Red LEDs when heart rate is in between 80 to 90 and Red LEDs of the cube when heart rate is greater than 90 .

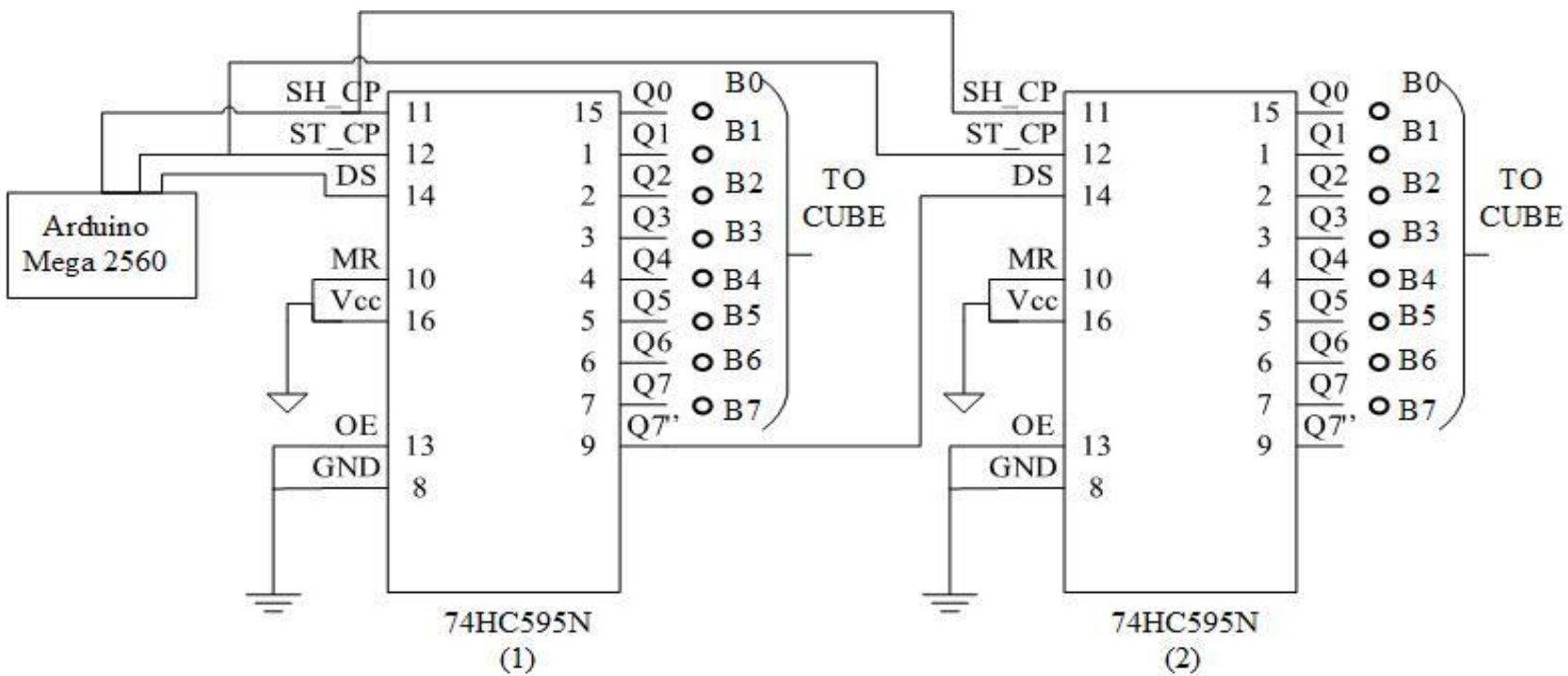

(1)

(2)

Fig .3: Two Shift Registers serially connected to one another

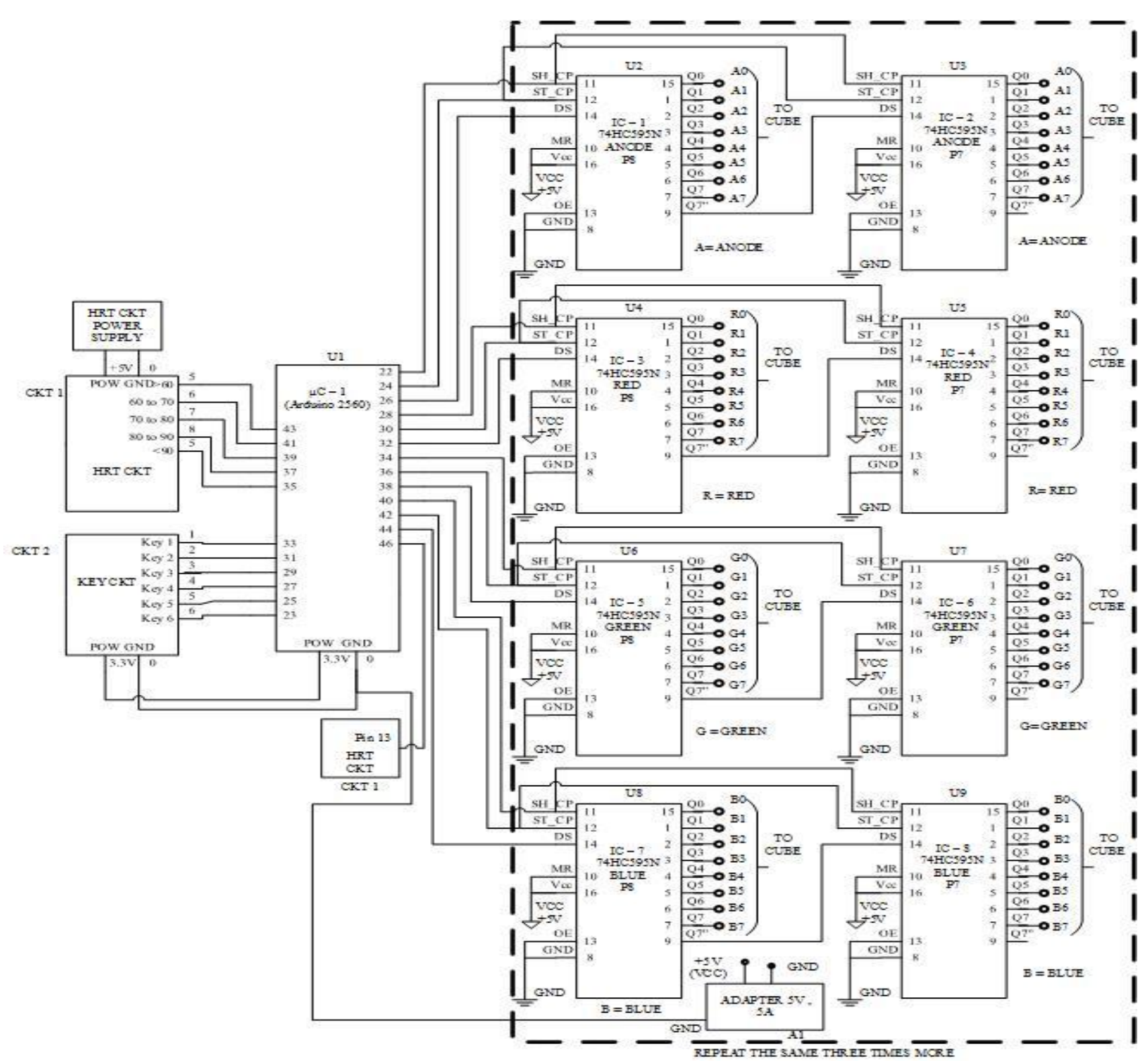

Fig. 4: Main cube circuit schematic 


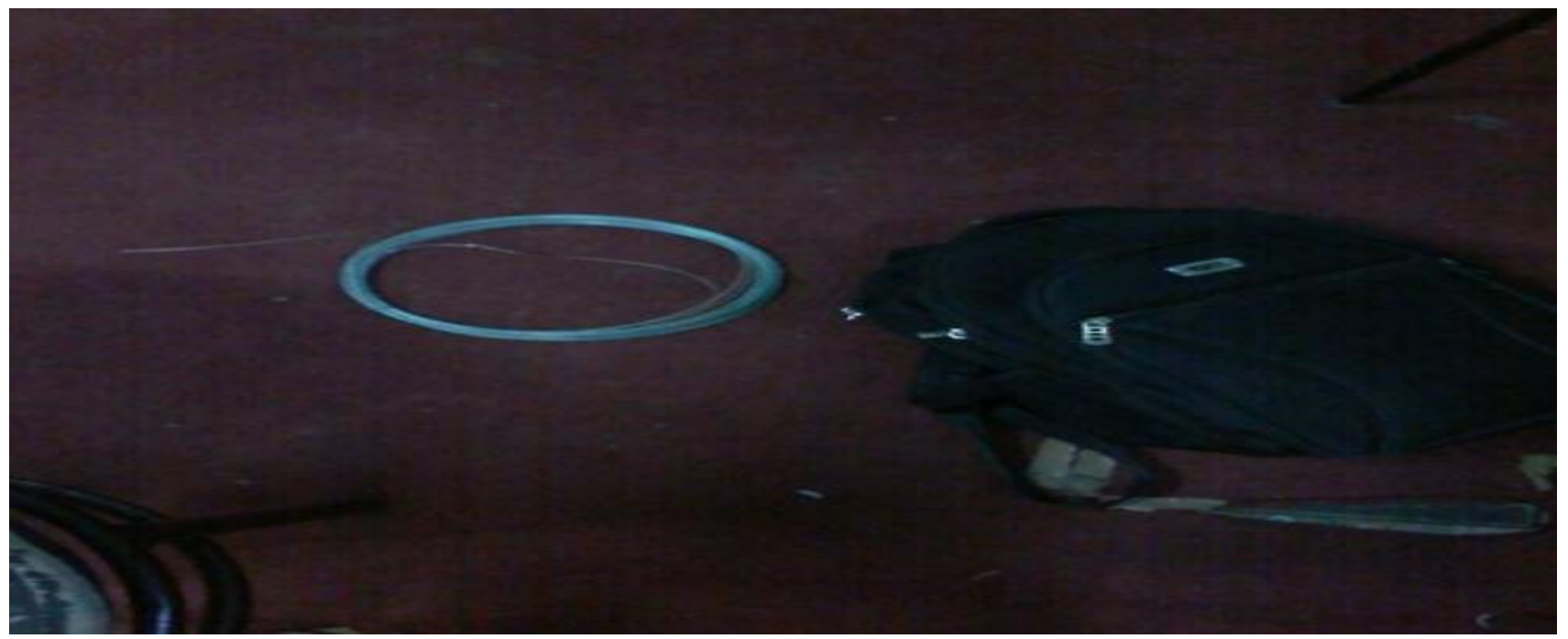

Fig. 5: Special Aluminium alloy wire

\section{Wires Used in Creating the Panels}

It is one of the most important parts of the output device. Proper selection of wire is must before creating a panel for RGB LED cube. Without the panels the cube cannot be created. It must meet the following specifications to get selected for this project:

- It must be a good conductor. If it is not then the electric current won't be able to pass through it.

- $\quad$ Strong in nature so that it can with stand the weight of 64 LEDs

- It should not have large cross section area otherwise it will provide high resistance to the current.

- It should be ductile in nature so that it can be moulded into any desired shape.

- $\quad$ It should be made up of such a material, so that it can be soldered with the RGB LEDs

The wire used in this project is made of aluminium alloy. The aluminium provides the electric conductivity to the wire. Some percentage of iron is also mixed in it so that it is strong in nature and withstands any kind of forces applied to it. It is very ductile in nature. These wires can be made straight through a torque force from drill machine. Straightening of wire is the most important part of panel building for RGB LED cube.

\section{Flow Charts}

\subsection{Flow Chart of Main Cube}

The Flow Chart of the main cube as mentioned in figure 6 shown the working of the software part of the main cube. It starts with reading of input from six switches. When key -1 is pressed Heart beat circuit gets activated and its following outputs are shown on the 3D RGB LED cube. First, the pattern generated is in accordance to the heart pulse. When heart pulse is HIGH, the entire LEDs of first panel glow and when heart pulse is LOW, en they are turned off. Therefore the LEDs blink in accordance to the heart pulse. Secondly, the colour of the LEDs is decided by the heart rate of the person. This is calculated by master controller of heart beat circuit. The different color of LEDs that glow in the RGB LED cube thus indicates the heart rate of the person. When we press key - 2, the whole RGB LED cube lights up in green colour. This indicates the proper functioning of all the LEDs of the cube. When key -3 is pressed, study patterns are shown on 3D RGB LED cube such as Square, Horizontal lines, Vertical lines, a point in space and 4 corners of a square. These patterns will help vari- ous students in learning different concepts regarding 1D, 2D and 3D space.

When key - 4 is pressed, a different study pattern is shown on the RGB LED cube. This is time the pattern tells us about the mathematical concepts of parallel lines, skew lines and intersecting lines. When key -5 is pressed, all the LEDs of the cube are turned off. This is needed in the system so that we can clear all the previous stored values of the shift registers and begin the system again from its initial conditions.

When key- 6 is pressed, the entertainment mode gets activated and cube shows various patterns for entertaining the people. For the cube to show any pattern it is important that one of these key is pressed. When a key is pressed, the corresponding pin of the micro controller goes high which calls the sub routine function built for that key.

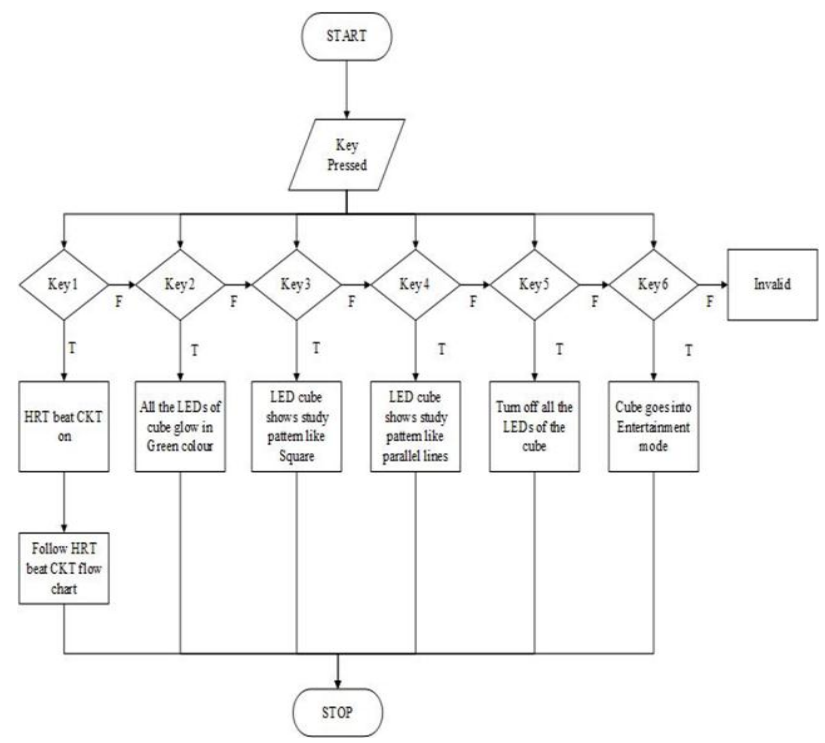

Fig. 6: Flow chart of main cube

\subsection{Flow Chart of Shift Register}

The Flow Chart of the Shift register as mentioned in figure 7 explains the working of all the Shift registers used in the cube. It starts with setting the latch pin of shift register low. Then a loop starts which is repeated for 8 times. Since we are sending a byte of data to the shift register used in the cube it is repeated 8 times. Inside the loop, the first step is to set the clock pin low. Now the first bit out of the 8 bit of data is sent on DS pin of the shift register. 
Now, the clock pin of shift register is set high. Thus the data on the DS pin gets stored inside the shift register. Since the data on DS pin is already stored in shift register, it is made low to accept the next bit of data. This is the point where the loop ends. To show the stored data on the output pins, we need to set the latch pin high. Thus the desired results are obtained on the output pins of shift register.

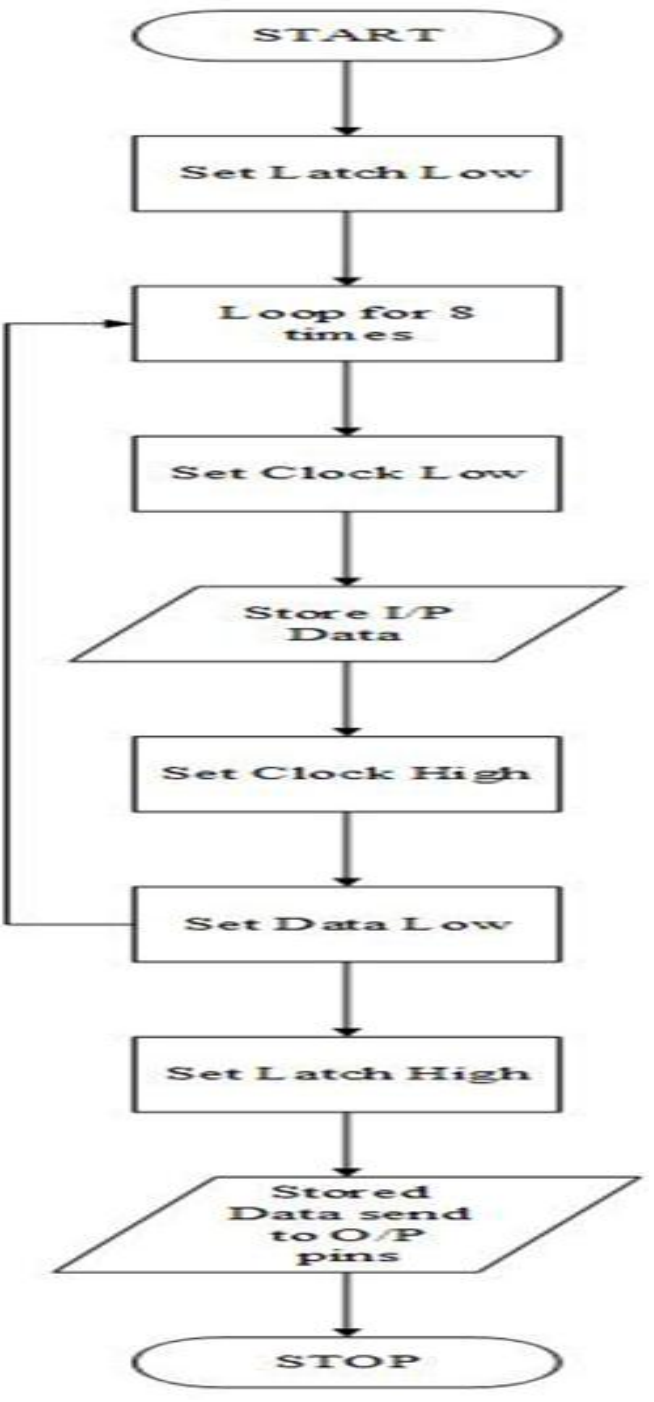

Fig. 7: Flow Chart of Shift Register

\section{Assembly and Testing}

The first part of testing for the project was done on a breadboard in which only 2 RGB LEDs were operated using a single shift register IC and Arduino UNO.

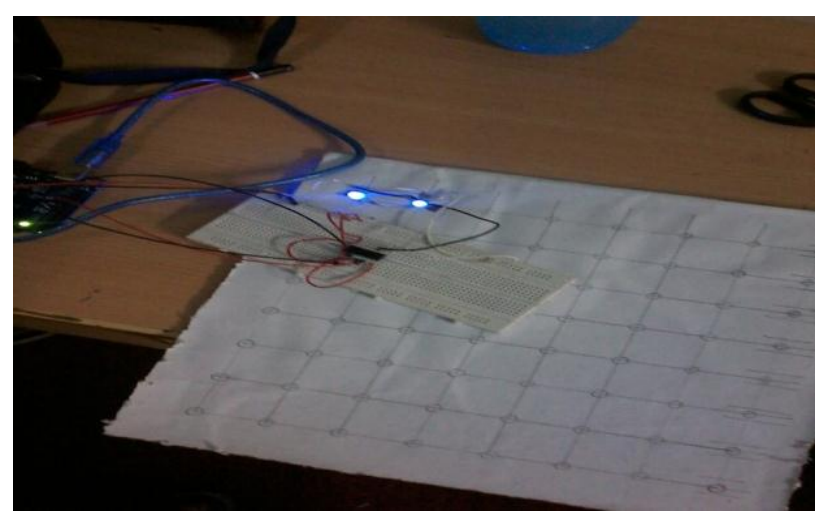

Fig. 8: Testing the RGB LEDs
The second part of testing for the project was also done on a breadboard in which one panel of RGB LEDs were operated using a two shift register IC and Arduino UNO.

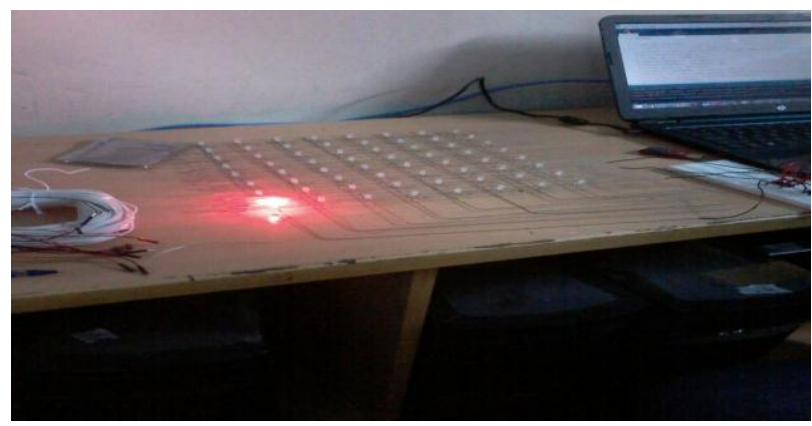

Fig. 9: Testing the RGB LEDs panel

The third part of testing for the project was also done after the assembly of the whole project as one instrument. The program code of the final project was adjusted and corrected according the system functionalities.

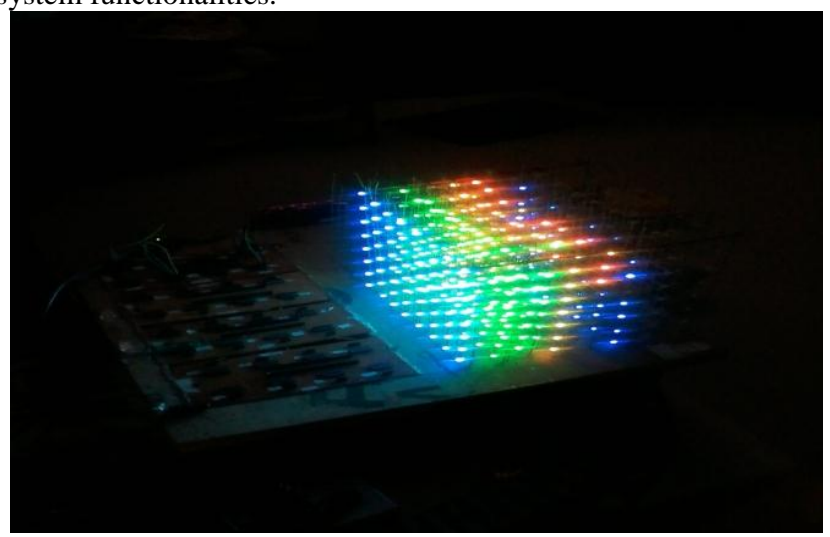

Fig. 10: Testing of the whole setup

Finally, the circuit was assembled and tested on zero PCB. It is implemented using three micro controllers (Arduino Mega 2560, Arduino UNO and AT89s52), Heart beat pulse sensor and shift registers $(74 \mathrm{HC} 595 \mathrm{~N})$.

\section{Results}

The main objective of the paper to design a 3D display device using RGB LEDs was fulfilled and implemented. The various modes (entertainment modes, study modes and heart beat mode) were also implemented in it.

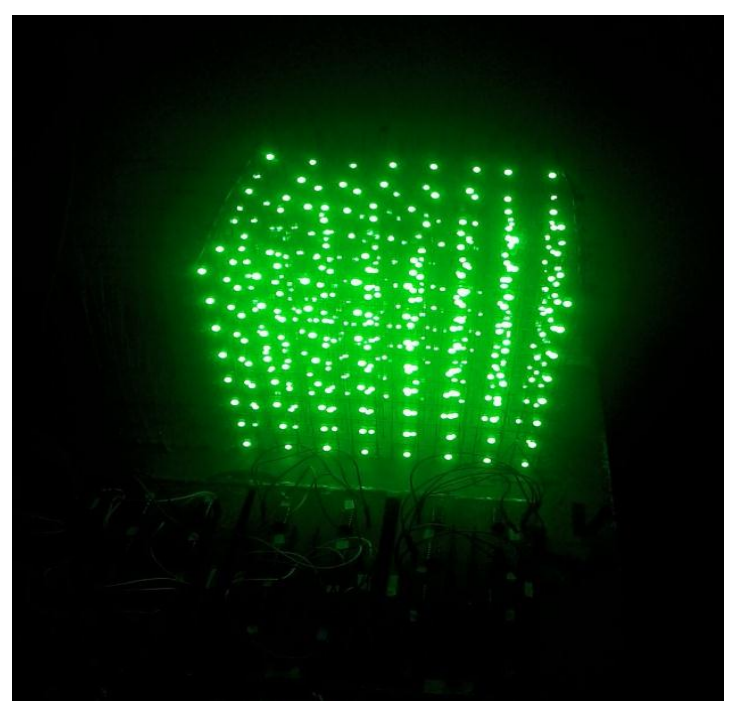

Fig. 11: 3 Dimentional Cube 


\section{Conclusion}

This paper concludes that a 3D RGB LED cube was designed using 512 RGB LEDs. The RGB LEDs were placed like a lattice structure. The basic motive was to implement entertainment mode, study mode and heart beat mode. The various issues regarding the study of 3D concepts in maths and chemistry were improved using the cube. Different patterns for entertainment, pleased the audience and through heart beat mode we could see the difference of heart rate according the color of RGB LEDs.

\section{References}

[1] Luke Ausley, Joshua Moyerman \& Andrew (2013), “3D LED Cube" senior design 1 Project documentation (Group - 15), University of Central Florida.

[2] Fangzheng Song, Nelson Lee, Candace Chen, Yang Liu \& Tristan Sturgess (2014),"True 3D image manipulator”, ELEC/CENG Design Project report (Group -16), Dept. Elect. And Cs. Eng., University of Victoria.

[3] David Wyatt \& Lawrence Wujanto (2005), "A Volumetric 3D LED Display", Final year Project , ,Introductory Digital Systems Laboratory, MIT, Cambridge.

[4] Katarína Žáková (2016), "The Use of 3D LED Cube for Basic Programming Teaching", IFAC-PapersOnLine 49, 6, 203-206. 